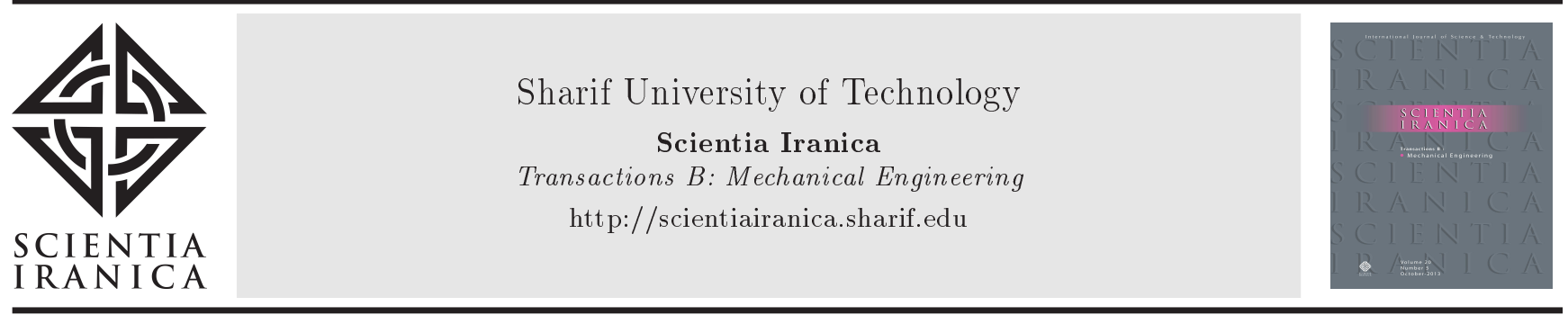

\title{
A class of synchronized nonlinear two-DOF systems with a closed-form solution
}

\author{
A. Soroushian ${ }^{a, *}$, G. Ahmadi ${ }^{b}$, and S. Amiri ${ }^{a}$ \\ a. Structural Engineering Research Center, International Institute of Earthquake Engineering and Seismology, Tehran 19537, Iran. \\ b. Department of Mechanical \& Aeronautical Engineering, Clarkson University, Potsdam, NY 13699-5725, USA.
}

Received 22 June 2018; received in revised form 12 August 2018; accepted 27 August 2018

\section{KEYWORDS}

Nonlinear dynamic;

Exact response;

Collision;

Static equilibrium;

Piece-wise linear;

Complexity.

\begin{abstract}
Nonlinear dynamic behavior of structural systems has a significant role in many engineering applications. Related analysis methods are typically numerical. Accordingly, to verify and test their accuracy, availability of nonlinear systems with exact closedform solutions is important. In this paper, a three-parameter system, with an exact analytical solution, consisting of two synchronized colliding mass-spring-dashpot systems, is introduced, and the validity of the exact solutions is demonstrated. Simplicity of the system and its exact response, and the capability to control the frequency content, as well as the severity of the oscillatory behavior are addressed as the main features of the introduced system. The potential future works are outlined.
\end{abstract}

(C) 2018 Sharif University of Technology. All rights reserved.

\section{Introduction}

Behaviors of structural systems are typically nonlinear and dynamic. In certain cases, nonlinear dynamic behavior can be simplified to linear dynamic, nonlinear static, or even linear static behaviors. For many applications, however, no simplification is plausible, and the full nonlinear dynamic behavior needs to be treated. Analysis of tall buildings or large bridges against seismic excitations is an example [1-5]. The general approach to analyzing structures' nonlinear dynamic behaviors involves:

1. Discretizing equations of motion as well as boundary and initial conditions, in space;

2. Solving the resulting initial value problem $[6,7]$.

For a typical nonlinear structural system, the semidiscretized problem is given as follows:

*. Corresponding author.

E-mail addresses: a.soroushian@iiees.ac.ir, \& aram.soroushian@gmail.com (A. Soroushian)

doi: $10.24200 /$ sci.2018.20891

$$
\begin{array}{ll}
\mathbf{M} \ddot{\mathbf{u}}(t)+\mathbf{f}_{\text {int }}(\mathbf{u}, t)=\mathbf{f}(t) \quad 0 \leq t<t_{\text {end }}, \\
\text { Initial conditions: } & \begin{array}{l}
\mathbf{u}(t=0)=\mathbf{u}_{0} \\
\dot{\mathbf{u}}(t=0)=\dot{\mathbf{u}}_{0} \\
\mathbf{f}_{i n t}(t=0)=\mathbf{f}_{i n t_{0}}
\end{array}
\end{array}
$$

Additional constraints: $\mathbf{Q}$.

In Eq. (1), $t$ stands for time; $t_{\text {end }}$ is the duration of analysis; $\mathbf{M}$ represents the mass matrix; $\mathbf{f}_{i n t}$ implies the internal force; $\mathbf{f}(t)$ is the excitation; $\mathbf{u}(t), \dot{\mathbf{u}}(t)$, and $\ddot{\mathbf{u}}(t)$ denote the displacement, velocity, and acceleration, respectively; $\mathbf{u}_{0}, \dot{\mathbf{u}}_{0}$, and $\mathbf{f}_{\text {int }_{0}}$ stand for the initial displacement, velocity, and internal force, respectively $[7,8] ; \mathbf{Q}$ represents additional restricting conditions in nonlinear problems, e.g., impact or elastic-plastic behavior $[9,10]$. For linear problems, $\mathbf{f}_{i n t}(\mathbf{u}, t)=\mathbf{K u}+\mathbf{C} \dot{\mathbf{u}}$, and $\mathbf{K}$ and $\mathbf{C}$ stand for the stiffness and damping matrices, respectively. Time integration is the most common approach to computing the responses of nonlinear initial value problems given by Eq. (1) [11,12]. However, similar to many other 
nonlinear analysis methods, time integration leads to numerical inaccuracy and inexact responses [1315]. Furthermore, in nonlinear structural dynamic analysis, the inaccuracy cannot be easily decreased in all analyses [8,13,15-17]. This is not in agreement with the essentiality in many practical approximate analyses, for which the accuracy needs to be sequentially improved [18-22].

Studying nonlinear structural dynamic behavior and analysis from different theoretical and practical points of view started about a century ago [23-25] and have continued more intensively over the last decades [26-38] and, particularly, the last years [39-43]. There is a common consensus that nonlinear analyses carried out with very small analysis parameters lead to exact responses (for instance, consider time integration analysis, for which the main analysis parameter is the integration step size [44]). However, "very small" is a problem-dependent notion [8], and implementation of excessively small analysis parameters may also produce significant round-off errors and is computationally expensive, generally [45]. The uncertainty in the notion of "very small" is intolerable for large problems with complex behavior. Therefore, the convention of assigning very small values to the algorithmic parameters and expecting sufficiently precise responses is neither practical nor reliable. Alternatively, implementation of an approach for evaluating errors with no need for the exact responses is reported in [8,46-48]. However, these error estimates will be reliable when the results converge properly $[8,34,45,47]$. Since proper convergence is too difficult to achieve in nonlinear problems $[8,13,16,17,44-49]$, availability of problems with exact responses remains important to assess the accuracy of the computational methods.

Assessment of accuracy and validation/ verification of nonlinear structural analysis studies is in close relation with errors and their convergence [50-53]. The error is given as follows:

$$
E_{\mathbf{u}}=\left\|\mathbf{u}^{\mathbf{a}}-\mathbf{u}\right\|
$$

where $\mathbf{u}^{\mathbf{a}}$ and $\mathbf{u}$, respectively, denote the approximately computed and exact displacement vectors [54], \| \| implies an arbitrary norm [55], and $E_{\mathbf{u}}$ stands for the displacement error (errors can be similarly defined for velocity, acceleration, and other responses).

The objective of this study is to introduce nonlinear systems with exact closed-form solutions, such that some of the important features can be set as desired, while the system and its response are kept as simple as possible. Availability of such systems would be of interest in the assessment of new computational methods $[48,56,57]$. For broader practical implementations, the features taken into account (in defining these systems) are mainly mathematical. The efforts reported in this paper extend the results reported in [57], considering decay of oscillations $[12,20]$, severity of oscillatory behavior (the number of oscillations throughout the analysis interval) [58], mathematical stiffness (the ratio of the largest to smallest periods in the response history) $[21,59]$.

The discussion is followed with introducing a simple linear SDOF (Single Degree Of Freedom) system. In view of the simplicity, the linear system is changed to a nonlinear SDOF system with exact closedform solution by adding an obstacle causing collision. The obstacle is then replaced with an additional mass considering structural symmetry [60,61]. Later the symmetry is sacrificed to obtain some desired features of the behavior. Several examples are presented in different parts of the discussion, and the paper is closed with a set of conclusions and potential future works.

\section{A three-parameter assembly of systems}

\subsection{Main idea}

The simple linear SDOF system is given as follows:

$$
\begin{array}{ll}
\ddot{u}+0.1 \dot{u}+u=0, & u(t=0)=1, \\
\dot{u}(t=0)=-0.05, & 0 \leq t<20,
\end{array}
$$

where the SI units are used. The exact response is given by Eq. (4) $[12,20]$ :

$$
\begin{aligned}
u(t) & =\left[\cos \omega_{D} t\right] e^{-0.05 t}, \quad \omega_{D}=\sqrt{0.9975} \\
0 & \leq t<20
\end{aligned}
$$

Herein, $e \cong 2.718281828459$. The corresponding displacement and velocity time histories are shown in Figure 1. By placing an obstacle in the static equilibrium position as shown in Figure 2, under the assumption of elastic collision (i.e., $\eta=1$, where $\eta$ is the coefficient of restitution $[62,63])$, the response becomes:
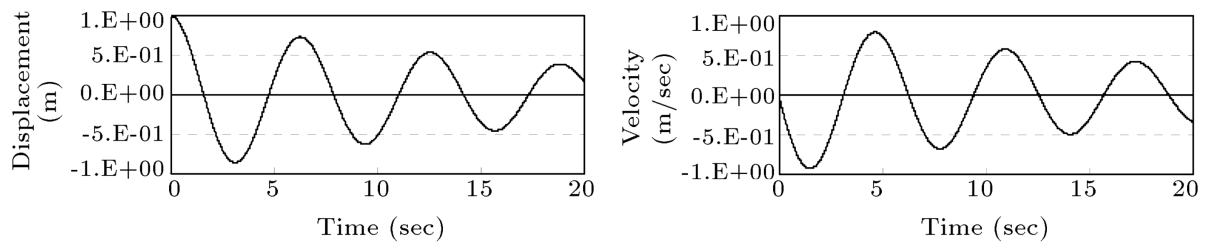

Figure 1. Exact response of the system given by Eq. (3). 


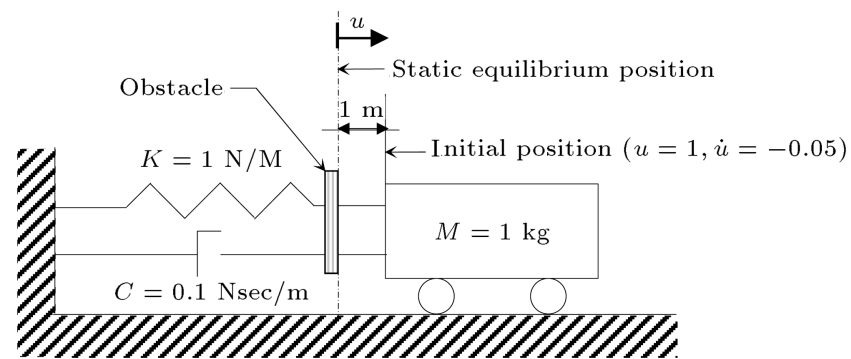

Figure 2. An SDOF system subjected to elastic collision to an obstacle in the static equilibrium position.

$$
\begin{aligned}
& u(t)=\left(\operatorname{sgn}\left[\cos \omega_{D} t\right]\right)\left[\cos \omega_{D} t\right] e^{-0.05 t}, \\
& \omega_{D}=\sqrt{0.9975}, \quad 0 \leq t<20,
\end{aligned}
$$

where "sgn" stands for the sign function defined as follows [64]:

$$
\operatorname{sgn}(x)= \begin{cases}-1 & \text { when } x<0 \\ 0 & \text { when } x=0 \\ 1 & \text { when } x>0\end{cases}
$$

The corresponding time variations of the response given by Eq. (5) are shown in Figure 3.

If, instead of an obstacle providing the elastic collision, the impacts resulted from another SDOF system, identical to the original system with reverse initial conditions, the motion of the coupled systems would be governed by:

$$
\begin{array}{ll}
\ddot{u}_{i}+0.1 \dot{u}_{i}+u_{i}=0, & i=1,2, \\
u_{i}(t=0)=(-1)^{i+1}, & \dot{u}_{i}(t=0)=0.05(-1)^{i}, \\
\eta=1, & 0 \leq t<20 .
\end{array}
$$

In view of the existing symmetry, the exact analytical expression for the response of Eq. (7) may be stated as follows:

$$
\begin{aligned}
& u_{1}(t)=\left(\operatorname{sgn}\left[\cos \omega_{D} t\right]\right)\left[\cos \omega_{D} t\right] e^{-0.05 t}, \\
& u_{2}(t)=-\left(\operatorname{sgn}\left[\cos \omega_{D} t\right]\right)\left[\cos \omega_{D} t\right] e^{-0.05 t}, \\
& \omega_{D}=\sqrt{0.9975}, \quad 0 \leq t<20 .
\end{aligned}
$$

Herein, $u_{1}$ and $u_{2}$ represent the displacements of the first and second masses, respectively. The corresponding displacement and velocity of masses 1 and 2 are displayed in Figure 4. It is seen that the time histories of displacement and velocity for mass 1 are identical to those shown in Figure 3 with the presence of an obstacle.

In the remainder of this section, the above system of coupled colliding oscillators is extended to a threeparameter system with the capability to control features such as mathematical stiffness. A corresponding sample response is shown in Figure 5. It is seen that
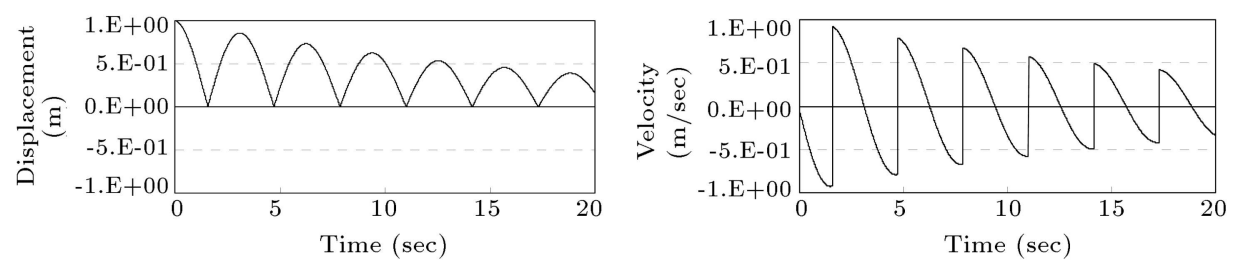

Figure 3. Exact response of the nonlinear system given in Figure 2.
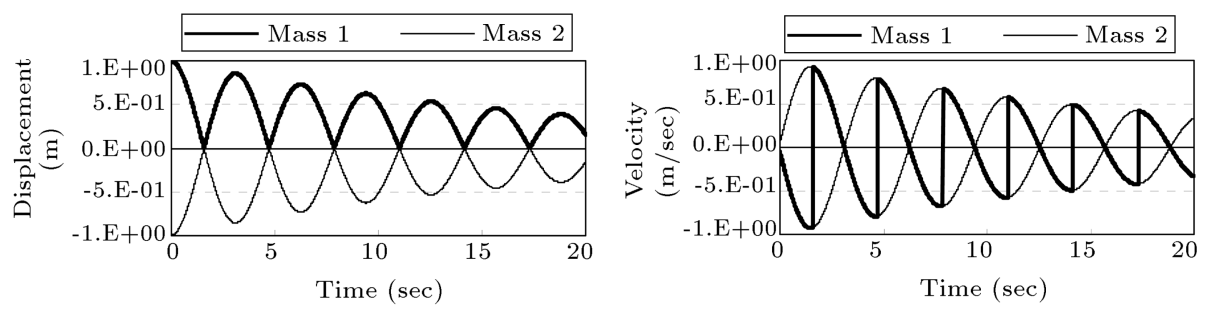

Figure 4. Exact response of the nonlinear system given by Eq. (7).

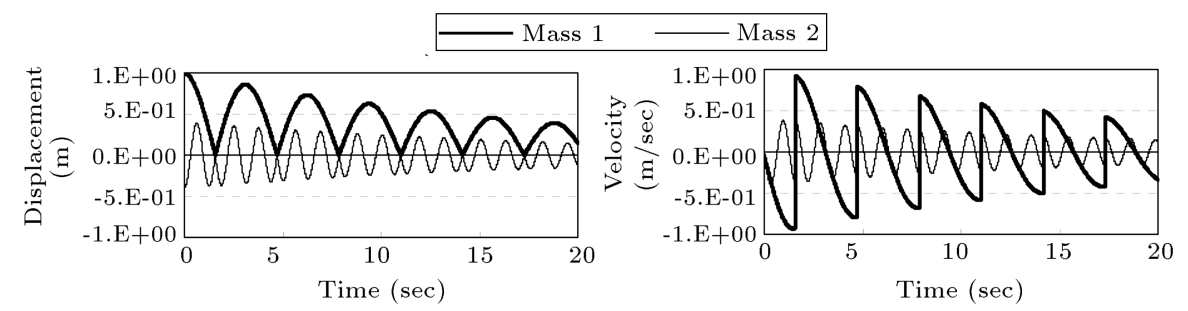

Figure 5. A sample extension of the exact response displayed in Figure 4. 
the collisions of the masses occur at selected common equilibrium point of the two masses at specific time instants.

\subsection{Detailed formulation}

To derive a generalized form of Eq. (7) and to cover a wider variety of structural systems, it is essential to understand the details of the process for developing the exact solution given by Eq. (8). With regard to the systems of colliding masses $[9,63]$, the main points are:

1. Characteristics of the two SDOF systems are set such that their natural frequencies are equal;

2. Characteristics of the two SDOF systems, including their initial conditions, are set such that the first collision occurs in the common static equilibrium position;

3. The impacts are elastic (the coefficient of restitution equals one [63]);

4. The characteristics of the two SDOF systems are set, such that, at the instant of the first collision, the momentums of the two masses are equal and opposite in sign;

5. The duration of the analysis and the viscous damping are set in such a way that the oscillations decay as desired;

6. The oscillations start from zero phases at peak displacements;

7. The masses are assumed concentrated at points.

Since the collisions are elastic and the two momentums are equal at the instant of collision, the velocities before the first collision simply change sign after the collision. Furthermore, since the natural frequencies are equal and the first collision occurs in the common static equilibrium position, the collisions continue to occur repeatedly in the static equilibrium position. The amplitudes, however, decrease due to damping, and this trend continues in time as predicted by Eq. (8).

To generalize the response shown in Figure 4 to that exemplified in Figure 5, we need to enforce the occurrence of the first collision of two masses in the common static equilibrium position, after a quarter of an oscillation period of the first mass, and a quarter and an integer number of oscillation periods of the second mass. Accordingly, since the momentums are equal and the collision is elastic, collision between the two masses will be repeated in time in the static equilibrium position, while the masses oscillate freely between the successive collisions. The "quarter period" noted above originates in the fact that, in Figure 4, the first collision occurs after one-fourth of the oscillation period (the "integer number" $N$ noted above is an arbitrary positive number). That is, Figure 5 indicates:

$$
\begin{aligned}
& \omega_{D_{2}}=(4 N+1)_{\infty} \omega_{D_{1}}, \\
& \omega_{D_{1}}={ }_{\infty} \omega_{1} \sqrt{1-\xi_{1}^{2}}, \quad N=0,1,2,3, \cdots .
\end{aligned}
$$

In Eq. (9), $\infty \omega_{D_{i}}$ represents the oscillatory frequency of mass $i(i=1,2)$ when the other mass is absent, $N$ is the above-mentioned integer number, and $\infty_{\infty} \omega_{1}$, which equals to one, stands for the undamped oscillatory frequency of Mass 1 when Mass 2 is absent. Equivalently, $N$ can be defined as the number of maximum displacements of the second mass, before the first collision. For example, Figures 4 and 5 , respectively, show $N=0$ and $N=1$. As implied in Eq. (9) and Figure 5, $N$ strongly affects the frequency content of the response. Sample simulation results for $N=1,2$, and 10 are shown in Figure 6. It is seen that the amplitude of the oscillation of the second mass decreases as $N$ increases. Figure 6 displays the Fourier amplitude of the displacements of the two masses [65]. Herein, different line thicknesses are used for different masses for clarity. Time steps of $0.001 \mathrm{~s}$ are used for accurate evaluation of the Fourier amplitudes. It is seen that as $N$ increases, the smallest period decreases, as in Eq. (9), and the corresponding Fourier amplitude decreases, too. Consistent with the earlier literature $[21,59,66-68]$, for the present system, the "mathematical stiffness ratio" is defined as follows:

$$
I_{m s}=\frac{\infty \omega_{D_{2}}}{\infty \omega_{D_{1}}}=4 N+1
$$

From the discussion of Figure 6 above, $I_{m s}$ is an upper bound of the ratio of the largest period (smallest frequency) non-trivially contributing to the response to the smallest period (largest frequency) non-trivially contributing to the response (see also $[21,59]$ ). In other words,

$$
\log (4 N+1) \geq \log \left(T_{L}\right)-\log \left(T_{S}\right) \Rightarrow I_{m s} \geq \frac{T_{L}}{T_{S}},
$$

where $T_{L}$ and $T_{S}$, respectively, denote the largest and smallest oscillatory periods non-trivially contributing to the response. Herein, $T_{L}$ can be approximated as:

$$
T_{L}=\pi / \omega_{D_{1}}=\pi / \sqrt{1-\xi_{1}^{2}}
$$

However, evaluation of $T_{S}$ requires numerical effort, especially for large values of $N$.

In order to ensure the equality of the momentums at the instant of collision, the condition:

$$
\xi_{1 \infty} \omega_{1}=\xi_{2 \infty} \omega_{2}
$$

is enforced that leads to the equality of the exponential terms in the solutions. Herein, $\xi_{i}$ and $\omega_{\infty} \omega_{i}$, respectively, stand for the damping coefficient and undamped 


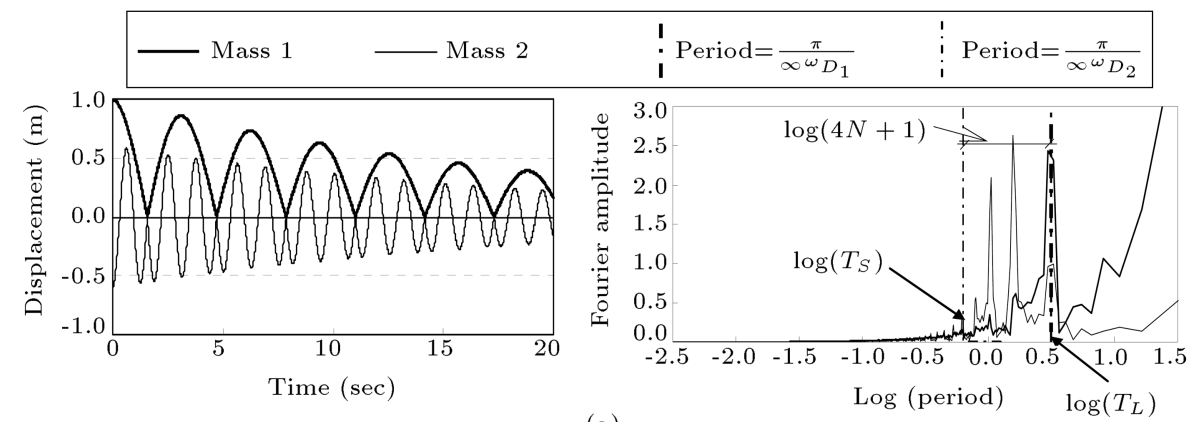

(a)
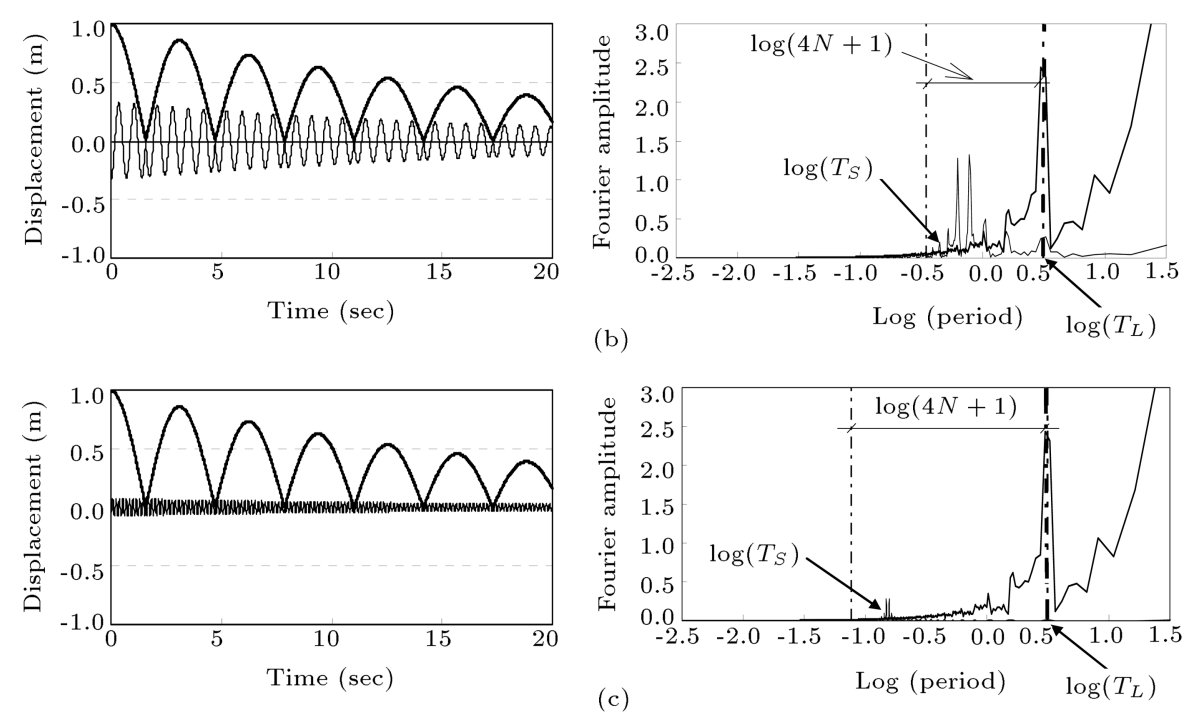

Figure 6. Extension of the responses displayed in Figure 4 in time and frequency domains: (a) $N=1$, (b) $N=2$, and (c) $N=10$.

natural frequency associated with mass $i(i=1,2)$. Consequently, the result of the momentums equality is stated below:

$$
m_{2}=-\frac{u_{1}(t=0)}{u_{2}(t=0)} \frac{m_{1}}{4 N+1} .
$$

In order to determine $\xi_{1}$ and $\xi_{2}$, it is assumed that:

$$
0 \leq \xi_{i=1,2}<1
$$

From Eqs. (10), (11), (14), and the relation of ${ }_{\infty} \omega_{D_{i}}=$ ${ }_{\infty} \omega_{i} \sqrt{1-\xi_{i}^{2}}[12,20]$, it is found that:

$$
\xi_{2}=\frac{\xi_{1}}{\sqrt{X^{2}-\xi_{1}^{2} X^{2}+\xi_{1}^{2}}},
$$

where:

$$
X=4 N+1
$$

In summary, when, the values of $u_{1}(t=0), m_{1}, \xi_{1}$, and $k_{1}$ are given, $m_{2} u_{2}(t=0), \xi_{2}$, and $k_{2}$ can be evaluated through Eqs. (12), (13), and (15). Then, the initial $\dot{u}_{2}$ $(t=0)$ can be obtained from:

$$
\dot{u}_{2}(t=0)=-\xi_{2 \infty} \omega_{2} u_{2}(t=0) .
$$

Based on main points 2 and 6 described in the beginning of this section, collisions, not in the static equilibrium position, are unacceptable. Therefore, the values of $m_{2}$ and $u_{2}(t=0)$ should be set so as to prevent the undesired collisions. A sufficiency requirement for the collision to occur at the static equilibrium point demonstrated in Appendix $\mathrm{A}$ is given as follows:

$$
0<-u_{2}(t=0) \leq \frac{3 u_{1}(t=0)}{4 N+1} .
$$

Because of the inequality sign in Eq. (18), there is redundancy for the selection of $u_{2}(t=0)$. Selection of smaller values for the initial displacement leads to smaller amplitudes for the oscillation of the second mass. Therefore, typically, the equality sign in Eq. (18) is used. The resulting one-parametric system of colliding oscillators may be stated as follows ( $N$ is the parameter):

$$
\begin{aligned}
& \ddot{u}_{1}+0.1 \dot{u}_{1}+u_{1}=0, \quad u_{1}(t=0)=1, \\
& \dot{u}_{1}(t=0)=-0.05, \\
& m_{2} \ddot{u}_{2}+2 m_{2 \infty} \omega_{2} \xi_{2} \dot{u}_{2}+k_{2} u_{2}=0,
\end{aligned}
$$




$$
\begin{aligned}
& u_{2}(t=0)=d_{2}, \quad \dot{u}_{2}(t=0)=-0.05 d_{2}, \\
& 0 \leq t<20, \\
& \eta=1, \quad m_{2}=\frac{1}{3}, \\
& \xi_{2}=\frac{0.05}{\sqrt{0.9975(4 N+1)^{2}+0.0025}}, \\
& k_{2}=\frac{0.9975(4 N+1)^{2}+0.0025}{3}, \\
& \infty \omega_{2}=\sqrt{\frac{k_{2}}{m_{2}}}, \quad d_{2}=\frac{-3}{4 N+1} .
\end{aligned}
$$

The corresponding exact response is given by:

$$
\begin{aligned}
u_{1}(t)= & \operatorname{sgn}(\cos [\sqrt{0.9975} t]) \cos [\sqrt{0.9975} t] e^{-0.05 t}, \\
u_{2}(t)= & \frac{-3}{4 N+1} \operatorname{sgn}(\cos [\sqrt{0.9975} t]) \\
& \cos [(4 N+1) \sqrt{0.9975} t] e^{-0.05 t} \\
0 \leq t< & 20 .
\end{aligned}
$$

These responses for different values of $N$ are displayed in Figure 7. For larger values of $N$, the ratio between the largest and smallest frequencies of oscillations increases, and the system may become mathematically more stiff $[21,59,66-68]$; similar trends are also shown in Figure 6. In other words, as $N$ increases, the oscillations of the second mass occur with higher frequencies and smaller amplitudes. Eqs. (15) and (16) show that an increase in $\xi_{1}$ causes an increase in $\xi_{2}$; therefore, an increase in the rate of the oscillations decays. Accordingly, $\xi_{1}$ is considered, as the second parameter of the proposed system. The resulting system is given by:

$$
\begin{aligned}
& \ddot{u}_{1}+2 \xi_{1} \dot{u}_{1}+u_{1}=0, \quad u_{1}(t=0)=1, \\
& \dot{u}_{1}(t=0)=-\xi_{1}, \\
& m_{2} \ddot{u}_{2}+2 m_{2 \infty} \omega_{2} \xi_{2} \dot{u}_{2}+k_{2} u_{2}=0 \text {, } \\
& u_{2}(t=0)=d_{2}, \quad \dot{u}_{2}(t=0)=-\xi_{1} d_{2}, \\
& 0 \leq t<20 \text {, } \\
& \eta=1, \quad m_{2}=\frac{1}{3}, \\
& \xi_{2}=\frac{\xi_{1}}{\sqrt{\left(1-\xi_{1}^{2}\right)(4 N+1)^{2}+\xi_{1}^{2}}}, \\
& k_{2}=\frac{\left(1-\xi_{1}^{2}\right)(4 N+1)^{2}+\xi_{1}^{2}}{3}, \\
& { }_{\infty} \omega_{2}=\sqrt{\frac{k_{2}}{m_{2}}}, \quad d_{2}=\frac{-3}{4 N+1},
\end{aligned}
$$
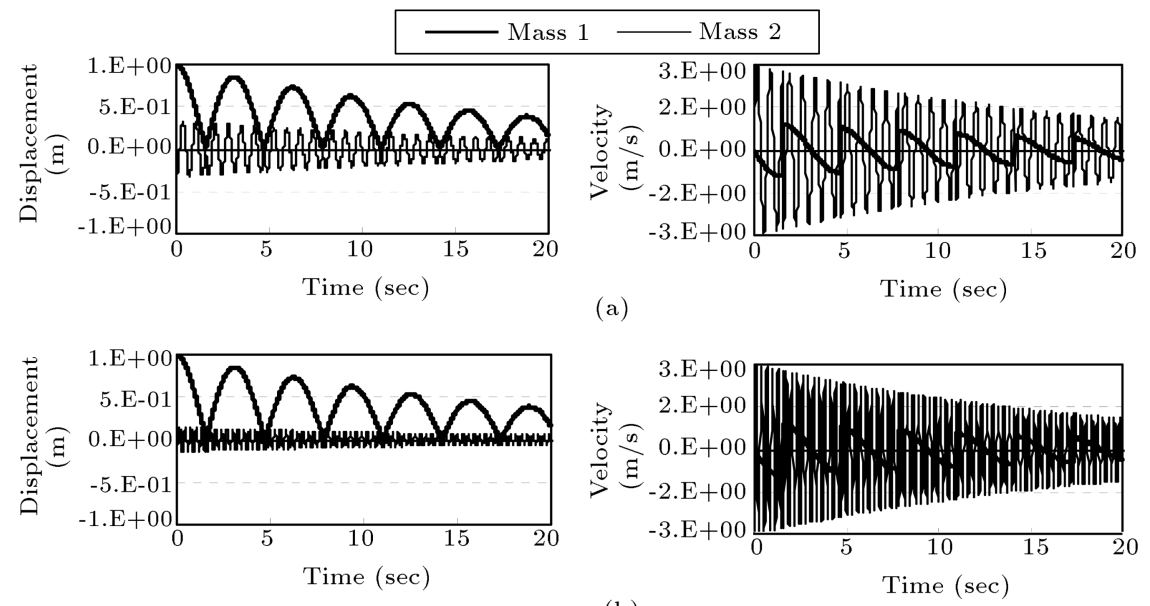

(b)
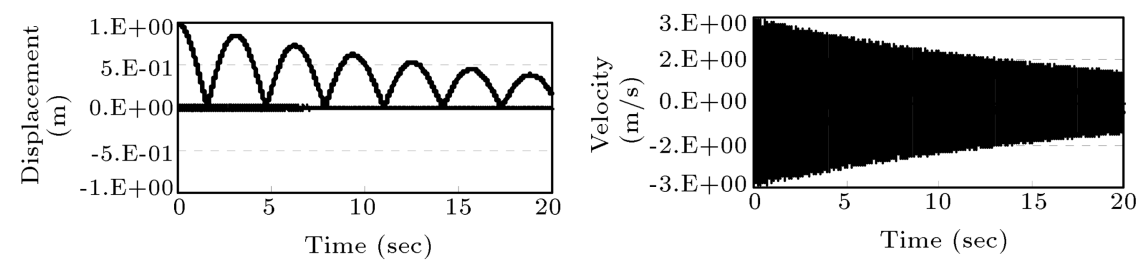

(c)

Figure 7. Exact response of the system given by Eq. (19): (a) $N=2$, (b) $N=5$, and (c) $N=20$. 
and the exact response is given as follows:

$$
\begin{aligned}
& u_{1}(t)= \operatorname{sgn}\left(\cos \left[\sqrt{1-\xi_{1}^{2}} t\right]\right) \cos \left[\sqrt{1-\xi_{1}^{2}} t\right] e^{-\xi_{1} t}, \\
& u_{2}(t)= \frac{-3}{4 N+1} \operatorname{sgn}\left(\cos \left[\sqrt{1-\xi_{1}^{2}} t\right]\right) \\
& \cos \left[(4 N+1) \sqrt{1-\xi_{1}^{2}} t\right] e^{-\xi_{1} t} \\
& 0 \leq t<20
\end{aligned}
$$

Figure 8 shows sample responses for different damping coefficients and various values of $N$. It is seen that the rate of decay increases as the damping coefficient increases. In addition, as $N$ increases, the frequency of oscillation of the second mass sharply increases.

The severity of oscillatory behavior is defined as how many oscillations occur in the duration under study $[57,58]$ and can be measured using an indicator:

$$
I_{O C}=\frac{t_{\text {end }}}{T_{S}} .
$$

For small values of $T_{S}, I_{O C}$ will be large, implying more oscillatory behavior. Hence, by setting the duration of the oscillations, $t_{\text {end }}$, equal to a multiple, $a_{t}$, of the duration used in Eq. (7), (i.e., $20 \mathrm{sec}$ ), it is assumed that:

$$
t_{\text {end }}=20 a_{t}, \quad a_{t}>0 .
$$

We can consider, $a_{t}$, as the third parameter (in addition to $N$ and $\xi_{1}$ ). In the resulting three-parameter system, the mathematical stiffness, the decay rate of oscillations, and the severity of the oscillatory can be controlled. Figure 9 shows sample solutions for fixed values of $N$ and $\xi_{1}$ and different values of $a_{t}$ where the behavior is more oscillatory when $a_{t}$ is larger. Consequently, by considering $N(N=0,1,2, \cdots), \xi_{1}$ $\left(0 \leq \xi_{1}<1\right)$, and $a_{t}\left(a_{t}>0\right)$, the three-parameter system can be defined as follows:

$$
\begin{aligned}
& \ddot{u}_{1}+2 \xi_{1} \dot{u}_{1}+u_{1}=0, \quad u_{1}(t=0)=1, \\
& \dot{u}_{1}(t=0)=-\xi_{1}, \\
& m_{2} \ddot{u}_{2}+2 m_{2 \infty} \omega_{2} \xi_{2} \dot{u}_{2}+k_{2} u_{2}=0
\end{aligned}
$$$$
u_{2}(t=0)=d_{2}, \quad \dot{u}_{2}(t=0)=-d_{2} \xi_{1}, \quad 0 \leq t<20 a_{t},
$$
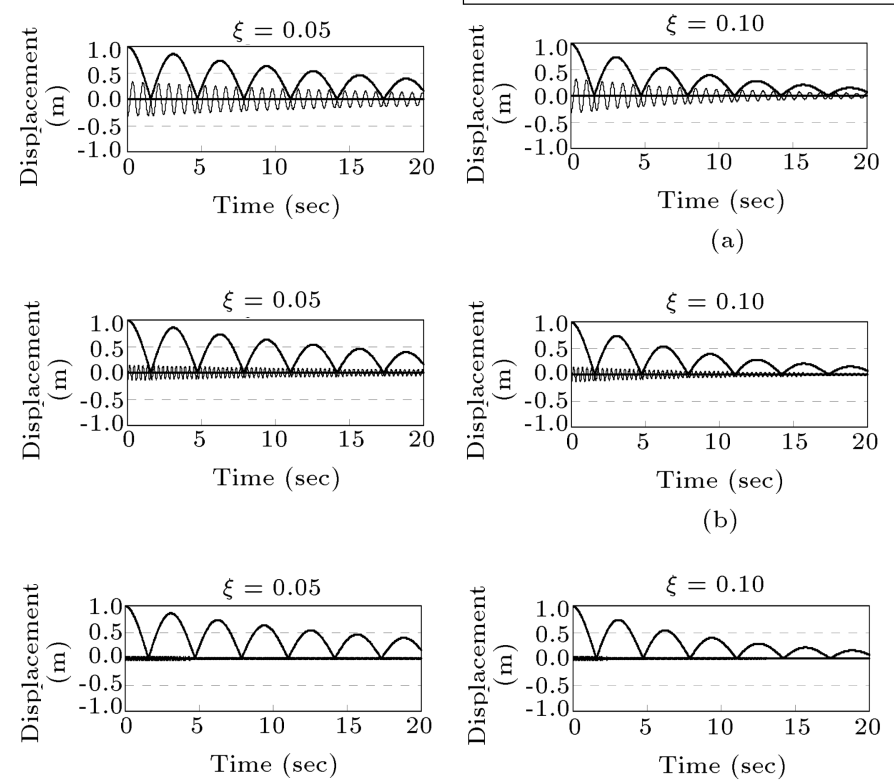

(c)

(a)

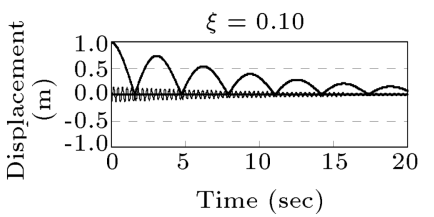

(b)
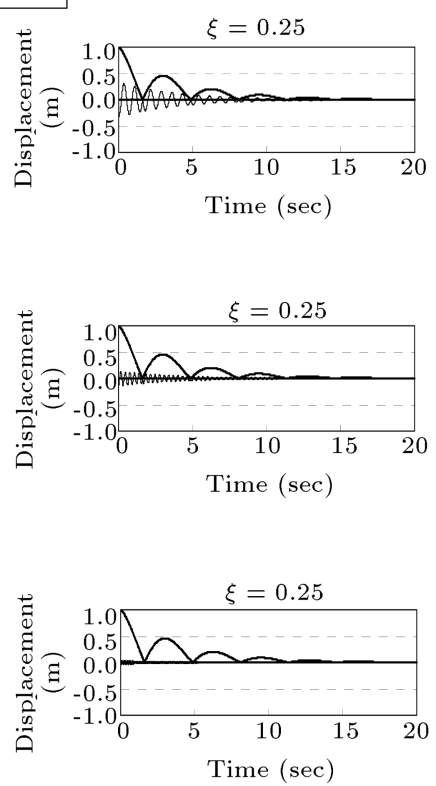

Time $(\mathrm{sec})$

Figure 8. Effects of $\xi_{1}$ on the displacements in Figure 7: (a) $N=2$, (b) $N=5$, and (c) $N=20$.

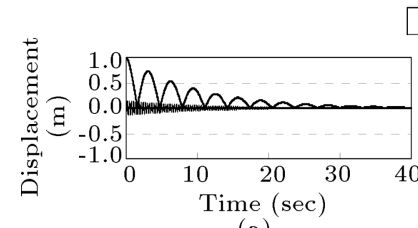

(a)

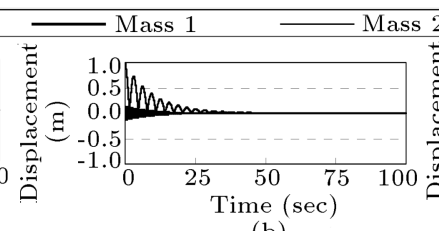

(b)

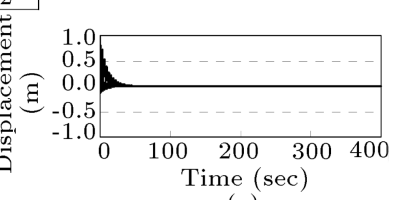

(c)

Figure 9. Effects of $a_{t}$ on the exact response in Figure 8(b), when $\xi_{1}=0.10$ : (a) $a_{t}=2$, (b) $a_{t}=5$, and (c) $a_{t}=20$. 


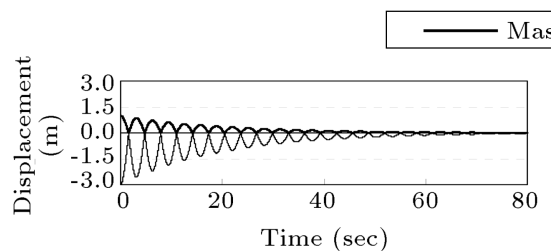

(a)

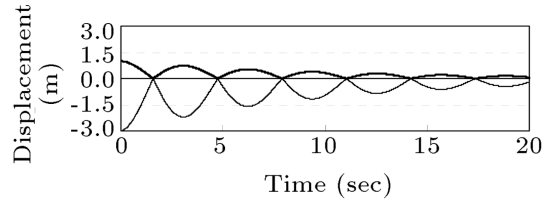

(c)

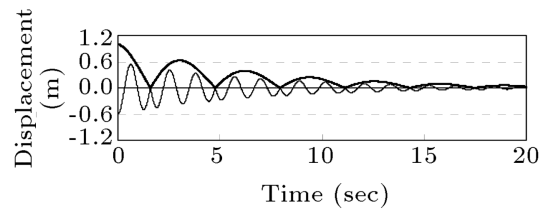

(e)

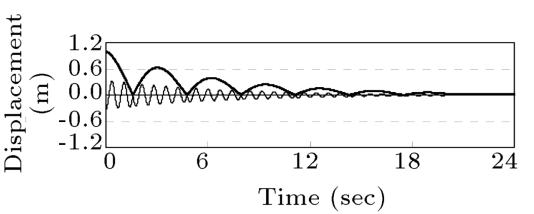

(g)

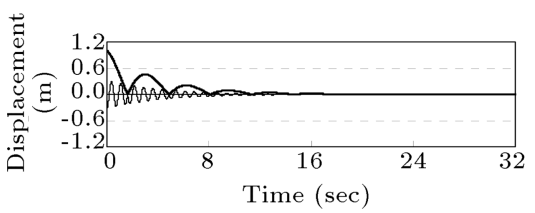

(i)

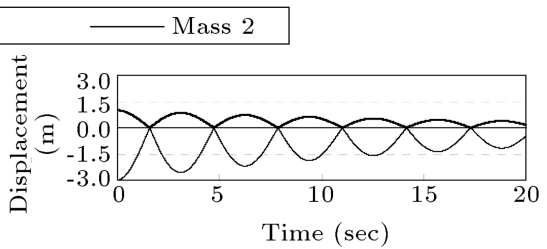

(b)

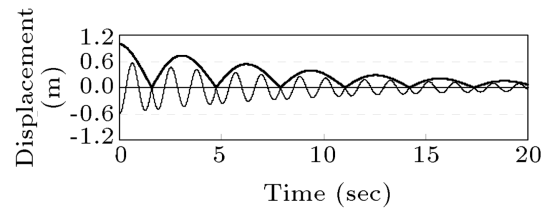

(d)

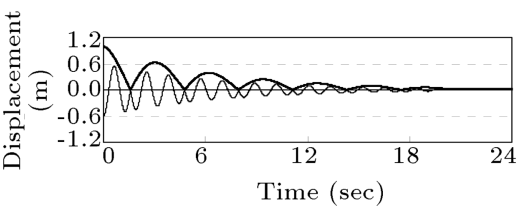

(f)

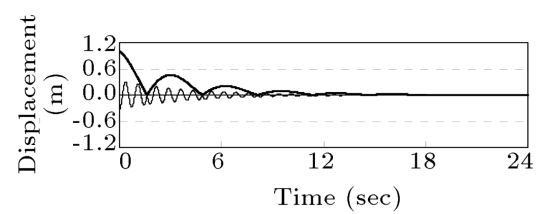

(h)

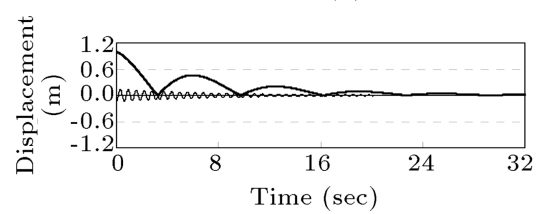

(j)

Figure 10. Examples for the exact responses of the three-parameter system introduced in Eq. (25): (a) $N=0, \xi_{1}=0.05$, $a_{t}=4$, (b) $N=0, \xi_{1}=0.05, a_{t}=1$, (c) $N=0, \xi_{1}=0.10, a_{t}=1$, (d) $N=1, \xi_{1}=0.10, a_{t}=1$, (e) $N=1, \xi_{1}=0.15$, $a_{t}=1$, (f) $N=1, \xi_{1}=0.15, a_{t}=1.2$, (g) $N=2, \xi_{1}=0.15, a_{t}=1.2$, (h) $N=2, \xi_{1}=0.25, a_{t}=1.2$, (i) $N=2, \xi_{1}=0.25$, $a_{t}=1.6$, and $(\mathrm{j}) N=5, \xi_{1}=0.25, a_{t}=0.8$.

$$
\begin{aligned}
& \eta=1, \quad m_{2}=\frac{1}{3} \\
& \xi_{2}=\frac{\xi_{1}}{\sqrt{\left(1-\xi_{1}^{2}\right)(4 N+1)^{2}+\xi_{1}^{2}}}, \\
& k_{2}=\frac{\left(1-\xi_{1}^{2}\right)(4 N+1)^{2}+\xi_{1}^{2}}{3}, \quad\left(\infty \omega_{2}=\sqrt{\frac{k_{2}}{m_{2}}}\right), \\
& d_{2}=\frac{-3}{4 N+1} .
\end{aligned}
$$

The exact response is given by:

$$
\begin{aligned}
u_{1}(t)= & \operatorname{sgn}\left(\cos \left[\sqrt{1-\xi_{1}^{2}} t\right]\right) \cos \left[\sqrt{1-\xi_{1}^{2}} t\right] e^{-\xi_{1} t}, \\
u_{2}(t)= & \frac{-3}{4 N+1} \operatorname{sgn}\left(\cos \left[\sqrt{1-\xi_{1}^{2}} t\right]\right) \\
& \cos \left[(4 N+1) \sqrt{1-\xi_{1}^{2}} t\right] e^{-\xi_{1} t}
\end{aligned}
$$

$$
0 \leq t<20 a_{t}
$$

In the examples shown in Figure 10, mathematical stiffness, severity of the oscillatory behavior, and decay of oscillations depend on $N, \xi_{1}$, and $a_{t}$. The values of the parameters used in Figure 10(a)-(j) clearly reveal a broad range of systems expressible by Eq. (25). In the first three figures, $N=0$ was used, while $N=1$ was selected in the next three figures and is followed by $N=2$ in Figure 10(g)-(i); finally, in Figure 10(j), the response for $N=5$ is shown. In Figure $10(\mathrm{a})$ and (b), the only difference is in the values of $a_{t}$. Similarly, with the exception of Figure $10(\mathrm{j})$, each two successive figures in Figure 10 differ in only one of the three parameters, $N, \xi_{1}$, and $a_{t}$. Figure $10(\mathrm{i})$ and $(\mathrm{j})$ differ in both $N$ and $a_{t}$.

It is worth comparing the three-parameter systems briefly introduced in this section with those presented in [57]. It should be pointed out that Eqs. (25) and (26) are somewhat different from those 
presented in [57]. The new three-parameter system and the associated response presented here are simpler to formulate and have a simpler physical interpretation. In addition, all the system's parameters are completely quantifiable.

\subsection{Responses validation}

The purpose in this section is to show that Eq. (26) is actually the response of the three-parameter system defined in Eq. (25). Since elastic collision between the two masses is the only source of nonlinearity in Eq. (25), the behavior can be considered piece-wise linear (see [34]). Consequently, the response exists and is unique (see $[21,69,70]$ ), and it is sufficient to show that Eq. (26) is a response of Eq. (25). By starting from the initial conditions, we can study the first segment of the piece-wise linear behavior with regard to simple structural dynamics principles $[12,20]$. Considering $t_{C_{1}}$ as the instant at which the first collision occurs, the responses at the first segment of the piece-wise linear behavior are given as follows:

$$
\begin{aligned}
& u_{1}(t)=\cos \left[\sqrt{1-\xi_{1}^{2}} t\right] e^{-\xi_{1} t}, \\
& u_{2}(t)=\frac{-3}{4 N+1} \cos \left[(4 N+1) \sqrt{1-\xi_{1}^{2}} t\right] e^{-\xi_{1} t}, \\
& 0 \leq t<t_{C_{1}} .
\end{aligned}
$$

It is followed by [65]:

$$
t_{C_{1}}=\frac{\pi}{2 \sqrt{1-\xi_{1}^{2}}}
$$

and:

$$
u_{1}\left(t_{C_{1}}\right)=u_{2}\left(t_{C_{1}}\right)=0 .
$$

Since the collision is elastic and occurs in the common static equilibrium position of the two masses, the collision mirrors (with respect to the time axis) the response that would have existed if there were no collision, as illustrated in Figure 11. Therefore:

$$
\begin{aligned}
u_{1}(t)= & \operatorname{sgn}\left(\cos \left[\sqrt{1-\xi_{1}^{2}} t\right]\right) \cos \left[\sqrt{1-\xi_{1}^{2}} t\right] e^{-\xi_{1} t}, \\
u_{2}(t)= & \frac{-3}{4 N+1} \operatorname{sgn}\left(\cos \left[\sqrt{1-\xi_{1}^{2}} t\right]\right) \\
& \cos \left[(4 N+1) \sqrt{1-\xi_{1}^{2}} t\right] e^{-\xi_{1} t} \\
0 \leq & t<t_{C^{*}}
\end{aligned}
$$

where:

$$
t_{C_{1}}<t_{C^{*}}=\min \left(\frac{\pi}{\sqrt{1-\xi_{1}^{2}}}, t_{C_{2}}\right),
$$
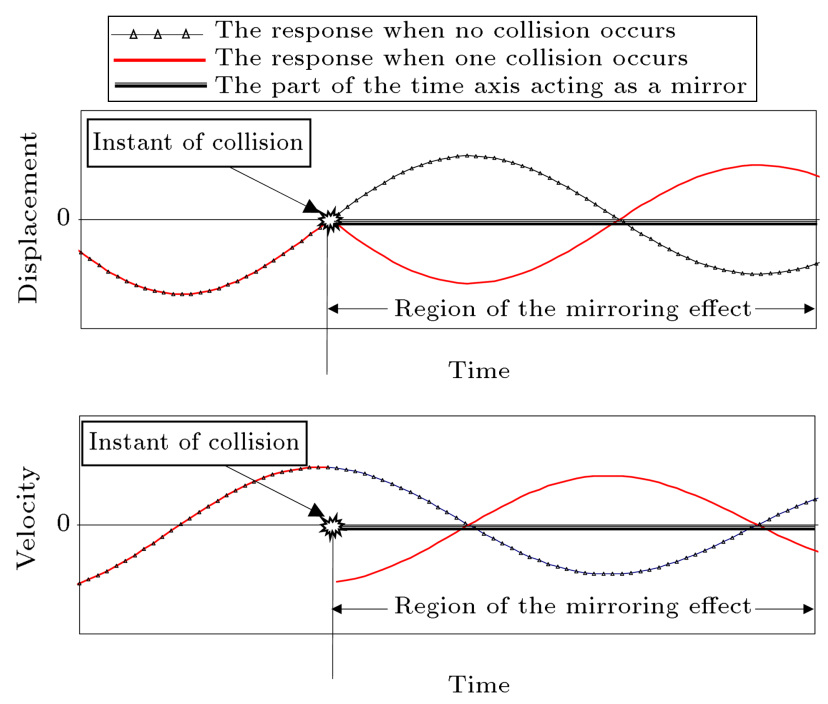

Figure 11. Typical effect of elastic collision between masses with equal momentum in their common static equilibrium position on each of the responses histories.

and $t_{C_{2}}$ stands for the instant of the second collision. Eq. (30) represents the two responses in all or a part of the second segment of the piece-wise linear behavior. Apparently, Eq. (30) does not imply any collision at the interval between $t_{C_{1}}$ and $\pi / \sqrt{1-\xi_{1}^{2}}$. Therefore, $t_{C^{*}}=\pi / \sqrt{1-\xi_{1}^{2}}$. Considering the changes of cosine when its argument equals $\pi$ and $1.5 \pi, \mathrm{Eq}$. (30) can be extended to:

$$
\begin{aligned}
u_{1}(t)= & \operatorname{sgn}\left(\cos \left[\sqrt{1-\xi_{1}^{2}} t\right]\right) \cos \left[\sqrt{1-\xi_{1}^{2}} t\right] e^{-\xi_{1} t} \\
u_{2}(t)= & \frac{-3}{4 N+1} \operatorname{sgn}\left(\cos \left[\sqrt{1-\xi_{1}^{2}} t\right]\right) \\
& \cos \left[(4 N+1) \sqrt{1-\xi_{1}^{2}} t\right] e^{-\xi_{1} t} \\
0 \leq & t<t_{C^{* *}}
\end{aligned}
$$

where:

$$
\frac{\pi}{\sqrt{1-\xi_{1}^{2}}}<t_{C^{* *}}=\min \left(\frac{3 \pi}{2 \sqrt{1-\xi_{1}^{2}}}, t_{C_{2}}\right) .
$$

Based on Eq. (32) and simple algebra, we get [65]:

$$
t_{C^{* *}}=\frac{3 \pi}{2 \sqrt{1-\xi_{1}^{2}}}=t_{C_{2}},
$$

and since, from Eqs. (32) and (33):

$$
u_{1}\left(t_{C_{2}}\right)=u_{2}\left(t_{C_{2}}\right)=0 \text {, }
$$

and the collisions are elastic, the trend displayed in Figure 11 is repeated. 
Once again, since cosine changes sign when its argument equals $1.5 \pi$, Eq. (32) can be extended as follows:

$$
\begin{aligned}
u_{1}(t)= & \operatorname{sgn}\left(\cos \left[\sqrt{1-\xi_{1}^{2}} t\right]\right) \cos \left[\sqrt{1-\xi_{1}^{2}} t\right] e^{-\xi_{1} t}, \\
u_{2}(t)= & \frac{-3}{4 N+1} \operatorname{sgn}\left(\cos \left[\sqrt{1-\xi_{1}^{2}} t\right]\right) \\
& \cos \left[(4 N+1) \sqrt{1-\xi_{1}^{2}} t\right] e^{-\xi_{1} t} \\
0 \leq & t<t_{C^{* * *}}
\end{aligned}
$$

where:

$$
\frac{3 \pi}{2 \sqrt{1-\xi_{1}^{2}}}<t_{C^{* * *}}=\min \left(\frac{2 \pi}{\sqrt{1-\xi_{1}^{2}}}, t_{C_{3}}\right) .
$$

According to Eq. (36), no collision occurs between the two masses at the interval between $t_{C_{2}}=3 \pi / 2 \sqrt{1-\xi_{1}^{2}}$ and $2 \pi / \sqrt{1-\xi_{1}^{2}}$; thus:

$$
t_{C^{* * *}}=\frac{2 \pi}{\sqrt{1-\xi_{1}^{2}}} .
$$

In view of Eqs. (36) and (38), Eq. (26) is valid at the time interval between $t=0$ and $t=2 \pi / \sqrt{1-\xi_{1}^{2}}$. Meanwhile, interestingly, the displacements and velocities of the two masses at $t=t_{C^{* * *}}$ are equal to those at $t=t_{C^{* * *}}$, when there are no collisions. This simply becomes apparent by comparing Eq. (27) with Eq. (36) at $t=t_{C^{* * *}}$. As an example, study results of the case of $N=1, \xi_{1}=0.1, a_{t}=1$ are reported in Figure 12 (the velocities are not plotted for the sake of brevity). Consequently, at $t=t_{C^{* * *}}$, we have returned to the starting stage of the discussion and Eq. (27), yet with smaller amplitudes. The discussion can, hence, be simply repeated, leading to the validity of Eq. (26) at the interval among $t=0, t=2 t_{C^{* * *}}=4 \pi / \sqrt{1-\xi_{1}^{2}}$, $t=0, t=3 t_{C^{* * *}}=6 \pi / \sqrt{1-\xi_{1}^{2}}$, etc. Accordingly, by repeating the discussion, Eq. (26) is valid for the desired time interval.

The second way to test the validity of Eq. (26) is through successful computations that converge to analytical solutions at the limit of zero values of the algorithmic parameters [71-73]. In other words, the numerical solutions of Eq. (25) should converge to those of Eq. (26).

\section{Conclusions}

With the aim of better evaluation of the accuracies that methods dedicated to nonlinear structural dynamic analysis provide, a class of three-parameter structural systems was introduced, whose exact analytical solutions were presented. Specifically,

1. The presented class consisted of two synchronized colliding mass-spring-dashpot systems oscillating on the same plane, with collisions between masses occurring in the common static equilibrium position;

2. The presented system and its exact analytical solution were simple;

3. The three parameters of these systems could be set for the desired mathematical stiffness, decay of oscillation, and severity of the oscillatory behavior;

4. Another interesting feature attributed to the proposed system was the piece-wise linear with an overall nonlinear behavior;
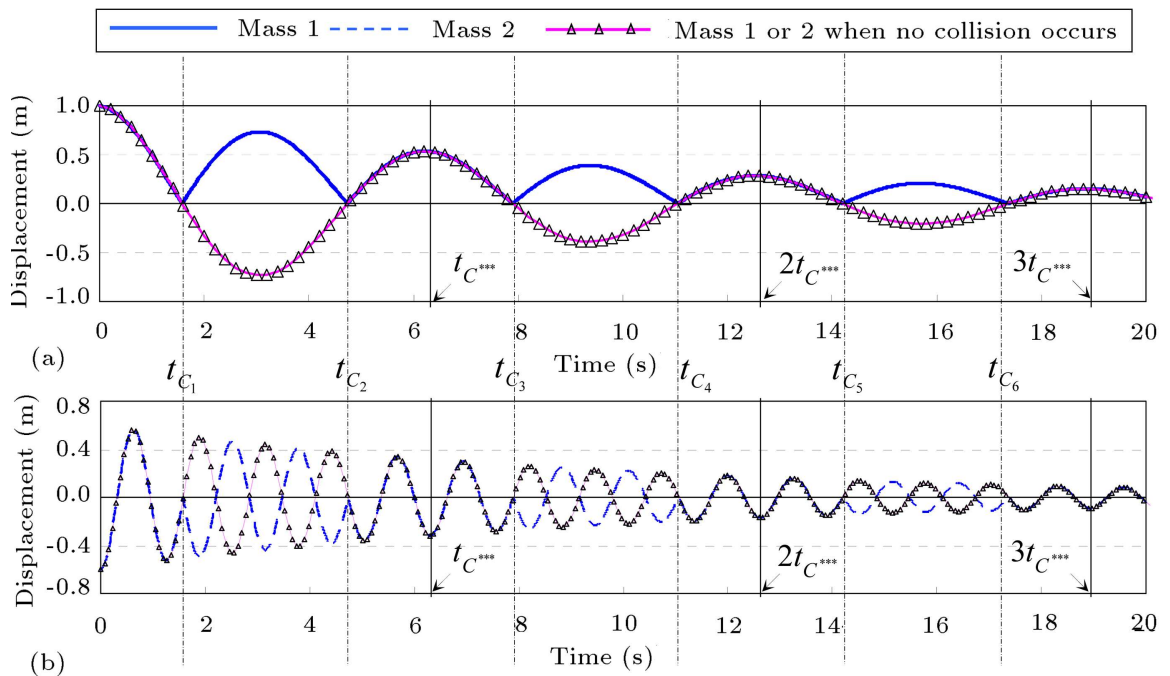

Figure 12. Coincidence at $t_{C^{* * *}}, 2 t_{C^{* * *}}$, and $3 t_{C^{* * *}}, \cdots$, of the actual responses with the responses obtained after disregarding the collisions, typically for the case $N=1, \xi_{1}=0.1, a_{t}=1$ : (a) The first mass, and (b) the second mass. 
5. The system can be used for assessment of accuracy in many engineering problems dealing with nonlinear behavior.

For the future work, we plan to extend the approach to a class of six-parameter nonlinear systems with exact closed-form solutions that provide additional flexibility for applications to real physical problems.

\section{References}

1. IBC, International Building Code. International Code Council, USA (2003).

2. Jeng, V. and Tzeng, W.L. "Assessment of seismic pounding hazard for Taipei city", Engineering Structures, 22(5), pp. 459-471 (2000).

3. Basoz, N.I., Kiremidjian, A.S., King, S.A., and Law, K.H. "Statistical analysis of bridge damage data from the 1994 Northridge, CA California", Earthquake Spectra, 15(1), pp. 25-53 (1999).

4. Hart, G.C. and Jain, A. "Performance-based wind evaluation and strengthening of existing tall concrete buildings in the Los Angeles region: dampers, nonlinear time history analysis and structural reliability", The Structural Design of Tall and Special Buildings, 23(16), pp. 1256-1274 (2014).

5. Sassi, M.A. "Nonlinear dynamic analysis of wind turbine towers subject to design wind and seismic loads", PhD Thesis, Colorado School of Mines, USA (2016).

6. Henrych, J., Finite Models and Methods of Dynamics in Structures, Elsevier, The Netherlands (1990).

7. Argyris, J. and Mlejnek, J.P., Dynamics of Structures, Elsevier, The Netherlands (1991).

8. Soroushian, A. "New methods to maintain responses' convergence and control responses' errors in the analysis of nonlinear dynamic models of structural systems", PhD Thesis, University of Tehran, Iran (2003) (in Persian).

9. Wriggers, P., Computational Contact Mechanics, John Wiley \& Sons, USA (2002).

10. Hughes, T.J.R., Pister, K.S., and Taylor, R.L. "Implicit-explicit finite elements in nonlinear transient analysis", Computer Methods in Applied Mechanics and Engineering, 17/18(1), pp. 159-182 (1979).

11. Chung, J. and Hulbert, G.M. "A family of singlestep Houbolt time integration algorithms for structural dynamics", Computer Methods in Applied Mechanics and Engineering, 118(1-2), pp. 1-11 (1994).

12. Chopra, A.K., Dynamics of Structures: Theory and Application to Earthquake Engineering, Prentice-Hall, USA (1995).

13. Soroushian, A., Wriggers, P., and Farjoodi, J. "On practical integration of semi-discretized nonlinear equations of motion. Part 1: reasons for probable instability and improper convergence", Journal of Sound and Vibration, 284(3-5), pp. 705-731 (2005).
14. Mohraz, B., Elghadamsi, F.E., and Chang, C.J. "An incremental mode superposition for non-linear dynamic analysis", Earthquake Engineering and Structural Dynamics, 20(5), pp. 471-481 (1991).

15. Nickell, R.E. "Nonlinear dynamics by mode superposition", Computer Methods in Applied Mechanics and Engineering, 7(1), pp. 107-129 (1976).

16. Rashidi, S. and Saadeghvaziri, M.A. "Seismic modeling of multispan simply-supported bridges using Adina", Computers \& Structures, 64(5-6), pp. 1025-1039 (1997).

17. Xie, Y.M. and Steven, G.P. "Instability, chaos, and growth and decay of energy of time-stepping schemes for nonlinear dynamic Equations", Communications in Numerical Methods in Engineering, 10(5), pp. 393-401 (1994).

18. NZS 1170.5:2004. "Structural design actions - Part 5: Earthquake actions", Standards New Zealand, New Zealand (2004).

19. Commentary NZS 1170.5:2004. "Structural design actions - Part 5: Earthquake actions", Standards New Zealand, New Zealand (2004).

20. Clough, R.W. and Penzien, J., Dynamics of Structures, McGraw-Hill, Singapore (1993).

21. Hairer, E. and Wanner, G., Solving Ordinary Differential Equations II: Stiff and Differential-Algebraic Problems, Springer, USA (1996).

22. Soroushian, A. and Amiri, S. "A comment on nonlinear time history analysis regulations of seismic code of New Zealand applicable in Eurocode 8 and many other seismic codes", 16th European Conference on Earthquake Engineering (16ECEE), Thessaloniki, Greece (2018).

23. Den Hartog, J.P. "Forced vibrations with combined coulomb and viscous damping", Transactions American Society of Mechanical Engineers, 53, pp. 107-115 (1931).

24. Wagstaff, J. "Experiments on the duration of impacts, mainly of bars with rounded ends, in elucidation of the elastic theory", Proceedings of the Royal Society of London Series A Containing Papers of a Mathematical and Physical Character, 105(733), pp. 544-570 (1924).

25. Leblanc, M. "Automatic balancer for rotating bodies", US Patent 1,159,052 (1915).

26. Wilson, E.L., Farhoomand, I., and Bathe, K.J. "Nonlinear dynamic analysis of complex structures", Earthquake Engineering and Structural Dynamics, 1(3), pp. 241-252 (1972).

27. Geradin, M., Idelsohn, S., and Hohhe, M. "Nonlinear structural dynamics via Newton and quasi-Newton methods", Nuclear Engineering and Design, 58(3), pp. 339-348 (1980).

28. Awrejcewicz, J. "Chaotic motion in a nonlinear oscillator with friction", KSME Journal, 2(2), pp. 104-109 (1988). 
29. Chen, X., Tamma, K.K., and Sha, D. "Virtualpulse time integral methodology: A new approach for computational dynamics. Part 2. Theory for nonlinear structural dynamics", Finite Elements in Analysis and Design, 20(3), pp. 195-204 (1995).

30. Esche, S.K., Kinzel, G.L., and Altan, T. "Issues in convergence improvement for non-linear finite element programs", International Journal for Numerical Methods in Engineering, 40(24), pp. 4577-4594 (1997).

31. Bathe, K.J. and Baig, M.M.I. "On a composite implicit time integration procedure for nonlinear dynamics", Computers and Structures, 83(31-32), pp. 2513-2524 (2005).

32. Gholampour, A.A. and Ghassemieh, M. "A weighted residual quadratic acceleration time integration method in nonlinear structural dynamics", $2010 \mathrm{Sec}-$ ond International Conference on Computer Research and Development, Kuala Lumpur, Malaysia (2010).

33. Wang, F. and Bajaj, A.K. "Nonlinear dynamics of a three-beam structure with attached mass and threemode interactions", Nonlinear Dynamics, 62(1-2), pp. 461-484 (2010).

34. Soroushian, A., Wriggers, P., and Farjoodi, J. "Practical integration of semi-discretized nonlinear equations of motion: proper convergence for systems with piecewise linear behavior", ASCE, Journal of Engineering Mechanics, 139(2), pp. 114-145 (2013).

35. Awrejcewicz, J., Krys'ko, V.A., and Vakakis, A.F., Nonlinear Dynamics of Continuous Elastic Systems, Springer, Germany (2004).

36. Bursi, O.S., Jia, C., Vulcan, L., Neild, S.A., and Wagg, D.J. "Rosenbrock-based algorithms and subcycling strategies for real-time nonlinear substructure testing", Earthquake Engineering and Structural Dynamics, 40(1), pp. 1-19 (2011).

37. Krenk, S. "Global format for energy-momentum based time integration in nonlinear dynamics", International Journal for Numerical Methods in Engineering, 100(6), pp. 458-476 (2014).

38. Kaveh, A., Fahimi-Farzam, M., and Kalateh-Ahani, M. "Performance-based multi-objective optimal design of steel frame structures: nonlinear dynamic procedure", Scientia Iranica, 22(2), pp. 373-387 (2015).

39. Rezaiee-Pajand, M. and Karimi-Rad, M. "A new explicit time integration scheme for nonlinear dynamic analysis", International Journal of Structural Stability and Dynamics, 16(9), pp. 1550054-1-1550054-26 (2016).

40. Alamatian, J. "Generalized implicit multi time step integration for nonlinear dynamic analysis", Scientia Iranica, 24(6), pp. 2776-2792 (2017).

41. Tae, D. and Tamma, K.K. "Mixed strong form representation particle method for solids and structures", Journal of Applied and Computational Mechanics, DOI: 10.22055/JACM.2018.24877.1216 (in Press).
42. Lee, T.Y., Chung, K.J., and Chang, H. "A new procedure for nonlinear dynamic analysis of structures under seismic loading based on equivalent nodal secant stiffness", International Journal of Structural Stability and Dynamics, 18(3), pp. 1850043-1-1850043-27 (2018).

43. Du, X., Yang, D., Zhou, J., Yan, X., Zhao, Y., and Li, S. "New explicit integration algorithms with controllable numerical dissipation for structural dynamics", International Journal of Structural Stability and Dynamics, 18(3), 1850044 (2018).

44. Bornemann, P.B., Galvanetto, U., and Crisfield, M.A. "Some remarks on the numerical time integration of non-linear dynamical systems", Journal of Sound and Vibration, 252(5), pp. 935-944 (2002).

45. Soroushian, A. "Proper convergence, a concept new in science and important in engineering", 4 th International Conference from Scientific Computing to Computational Engineering (4th IC-SCCE), Athens, Greece (2010).

46. Soroushian, A., Wriggers, P., and Farjoodi, J. "Asymptotic upper-bounds for the errors of Richardson extrapolation with practical application in approximate computations", International Journal for Numerical Methods in Engineering, 80(5), pp. 565-595 (2009).

47. Soroushian, A. "Pseudo convergence and its implementation in engineering approximate computations", 4th International Conference from Scientific Computing to Computational Engineering (4th IC-SCCE), Athens, Greece (2010).

48. Liu, S. and Valkó, P.P. "Optimization of spacing and penetration ratio for infinite-conductivity fractures in unconventional reservoirs: A section-based approach", SPE Journal, 22(6), pp. 1877-1892 (2017).

49. Low, K.H. "Convergence of the numerical methods for problems of structural dynamics", Journal of Sound and Vibration, 150(2), pp. 342-349 (1991).

50. Vassault, A., Hulin, A., Chapuzet, E., Arnaud, J., and Giroud, C. "Verification/validation of the performances of analytical method", Ann. Biol. Clin., 68(Spec no 1), pp. 247-294 (2010).

51. Roache, P.J. "Verification of codes and calculations", AIA A Journal, 36(5), pp. 696-702 (1998).

52. Petri, L.A., Sartori, P., Rogenski, J.K., and de Souza, L.F. "Verification and validation of a direct numerical simulation code", Computer Methods in Applied Mechanics and Engineering, 291, pp. 266-279 (2015).

53. Doebling, S.W., Hemez, F.M., Robertson, A.N., Maupin, R.D., Schultze, J.F., Cundy, A.L., and Hylock, J.E. "Validation of the transient structural response of a threaded assembly: Phase I", LA-14104MS, Los Alamos National Lab (LANL), Los Alamos, USA (2004).

54. Ralston, A. and Rabinowitz, P., First Course in Numerical Analysis, McGraw-Hill, USA (1978).

55. Noble, B. and Daniel, J.W., Applied Linear Algebra, Prentice-Hall, USA (1977). 
56. Mostaghel, N. and David, T. "Representations of coulomb friction for dynamic analysis", Earthquake Engineering and Structural Dynamics, 26(5), pp. 541548 (1997).

57. Soroushian, A. and Ahmadi, G. "A three parameter nonlinear dynamic system with exact closed form solution", 17th International Congress on Sound \& Vibration, Cairo, Egypt (2010).

58. Petzold, L.A., Jay, L.O., and Yen, J. "Numerical solution of highly oscillatory ordinary differential equations", Acta Numerica, 6, pp. 437-483 (1997).

59. Quateroni, A., Sacco, R., and Saleri, F., Numerical Mathematics, Springer, USA (2000).

60. Norris, C.H., Wilbur, J.B., and Utku, S., Elementary Structural Analysis, McGraw Hill, USA (1976).

61. Hsieh, Y.Y., Elementary Theory of Structures, 2nd Ed., Prentice-Hall, Englewood Cliffs, USA (1982).

62. Kuwabara, G. and Kimitoshi, K. "Restitution coefficient in a collision between two spheres", Japanese Journal of Applied Physics, 26(8), pp. 1230-1233 (1987).

63. Ruedigger, H., Christian, G. and Carsten, P. "Comparison 12: Collision processes in rows of spheresdefinition and ACSL solution", SNE-Simulation News Europe, 9(27), pp. 36-38 (1999).

64. Apostol, T.M., Calculus, I, John Wiley \& Sons, USA (1967).

65. Ayres, F. and Mendelson, E., Schaum's Outline of Calculus, McGraw-Hill, New York, USA (1999). Also http://mathworld.wolfram.com/

66. http://en.wikipedia.org/wiki/Stiff_equation

67. Bass, J.M. and Oden, J.T. "Numerical solution of the evolution equations of damage and rate-dependent plasticity", International Journal of Engineering Science, 26(7), pp. 713-740 (1988).

68. Ilie, S. and Gholami, S. "Simplifying stochastic mathematical models of biochemical systems", Applied Mathematics, 4(1A), pp. 248-256 (2013).

69. Soroushian, A., Arghavani, M., Rajabi, M., Saaed, A., and Sharifpour, M.M. "A proposition on the uniqueness of solutions for nonlinear structural dynamic models", 10th Biennial International Conference on Vibration Problems, Technical University of Liberec, Prague, Czech Republic (2011).

70. Collatz, L., The Numerical Treatment of Differential Equations, Springer, Berlin, Germany (1960).

71. Henrici, P., Discrete Variable Methods in Ordinary Differential Equations, John Wiley, New York, USA (1962).

72. Strikwerda, J.C., Finite Difference Schemes and Partial Differential Equations, Wadsworth \& Books/Cole, Pacific Grove, CA, USA (1989).

73. Gear, C.W., Numerical Initial Value Problems in Ordinary Differential Equations, Prentice Hall, Upper Saddle River, NJ, USA (1971).
74. Kachenovsky, M., Kolyagin, Y., Kutasov, A., Lukankin, G., Yakovlev, G., and Oganesyan, V., Geometry (A Textbook for Technical Schools), Mir Publishers, Russia (1982).

\section{Appendix A}

The aim here is to demonstrate that the condition given by Eq. (18) is sufficient to prevent collisions occurring at any other point except the static equilibrium position of the system defined in Section 2. According to Eqs. (7) and (8), the responses of the two masses may be stated as:

$$
\begin{aligned}
\begin{aligned}
& u_{1}(t)= u_{1}(t=0) \operatorname{sgn}\left(\cos \left[\sqrt{1-\xi_{1}^{2}} t\right]\right) \\
& \cos \left[\sqrt{1-\xi_{1}^{2}} t\right] e^{-\xi_{1} t}, \\
& u_{2}(t)= u_{2}(t=0) \operatorname{sgn}\left(\cos \left[\sqrt{1-\xi_{1}^{2} t}\right]\right) \\
& \cos \left[(4 N+1) \sqrt{1-\xi_{1}^{2}} t\right] e^{-\xi_{1} t}, \\
& u_{1}(t=0)>0 .
\end{aligned}
\end{aligned}
$$

It should be shown that, for the response in Eq. (A.1):

$$
u_{1}(t)>u_{2}(t)
$$

if:

$$
\begin{aligned}
& N=1,2,3, \cdots, \quad 0 \leq \xi_{1}<1, \\
& 0<-u_{2}(t=0) \leq \frac{3 u_{1}(t=0)}{4 N+1}, \\
& t \neq \frac{0.5 \pi}{\sqrt{1-\xi_{1}^{2}}}, \frac{1.5 \pi}{\sqrt{1-\xi_{1}^{2}}}, \cdots \quad \text { (Instants of } \\
& \text { collision at the static equilibrium position). }
\end{aligned}
$$

The first, second, and forth relationships in Eq. (A.3) are considered valid; the validity of the third relation is discussed (which is Eq. (18)). We first study the case in which the equality holds. That is:

$$
0<-u_{2}(t=0)=\frac{3 u_{1}(t=0)}{4 N+1} .
$$

Using Eq. (A.4), in Eq. (A.2), $u_{1}(t)$ and $u_{2}(t)$ may be restated as follows:

$$
u_{1}(t)=\operatorname{sign}\left(\cos \left[\sqrt{1-\xi_{1}^{2}} t\right]\right) \cos \left[\sqrt{1-\xi_{1}^{2}} t\right] e^{-\xi_{1} t},
$$




$$
\begin{aligned}
u_{2}(t)= & \frac{-3}{4 N+1} \operatorname{sign}\left(\cos \left[\sqrt{1-\xi_{1}^{2}} t\right]\right) \\
& \cos \left[(4 N+1) \sqrt{1-\xi_{1}^{2}} t\right] e^{-\xi_{1} t}, \quad u_{1}(t=0)>0 .
\end{aligned}
$$

Since the arguments of the two Sgn functions in Eq. (A.5) are identical, the following two ranges are studied separately:

$$
\begin{aligned}
& 2 k \pi-\frac{\pi}{2}<\sqrt{1-\xi_{1}^{2}} t<2 k \pi+\frac{\pi}{2}, \\
& (2 k+1) \pi-\frac{\pi}{2}<\sqrt{1-\xi_{1}^{2}} t<(2 k+1) \pi+\frac{\pi}{2}, \\
& \quad k=0,1,2,3 \cdots .
\end{aligned}
$$

For the first case, Eq. (A.5) will be simplified to:

$$
\begin{aligned}
& u_{1}(t)=\cos \left[\sqrt{1-\xi_{1}^{2}} t\right] e^{-\xi_{1} t}, \\
& u_{2}(t)=\frac{-3}{4 N+1} \cos \left[(4 N+1) \sqrt{1-\xi_{1}^{2}} t\right] e^{-\xi_{1} t}, \\
& u_{1}(t=0)>0 .
\end{aligned}
$$

By replacing $t$ with $\bar{t}$ defined as:

$$
2 k \pi+\bar{t}=\sqrt{1-\xi_{1}^{2}} t
$$

and using simple trigonometry [65], Eqs. (A.7) become:

$$
\begin{aligned}
u_{1}(t) & =\cos \bar{t} e^{-\xi_{1}\left(\frac{2 k \pi}{\sqrt{1-\xi_{1}^{2}}}+\frac{\bar{t}}{\sqrt{1-\xi_{1}^{2}}}\right)}, \\
u_{2}(t) & =\frac{-3}{4 N+1} \cos [(4 N+1) \bar{t}] e^{-\xi_{1}\left(\frac{2 k \pi}{\sqrt{1-\xi_{1}^{2}}}+\frac{\bar{t}}{\sqrt{1-\xi_{1}^{2}}}\right),} \\
-\frac{\pi}{2} & <\bar{t}<\frac{\pi}{2} .
\end{aligned}
$$

By using Eq. (A.9), the validity condition (A.2) (in the first case of Eq. (A.6) is equivalent to the validity of the following:

$$
D(\bar{t})>0, \quad-\frac{\pi}{2}<\bar{t}<\frac{\pi}{2},
$$

where:

$$
D(\bar{t})=u_{1}(\bar{t})-u_{2}(\bar{t}) .
$$

From Eqs. (A.9) and (A.11):

$$
\begin{aligned}
D(\bar{t})= & e^{-\xi_{1}\left(\frac{2 k \pi}{\sqrt{1-\xi_{1}^{2}}}+\frac{\bar{t}}{\sqrt{1-\xi_{1}^{2}}}\right)}\left(\cos \bar{t}+\frac{3}{4 N+1}\right. \\
& \cos [(4 N+1) \bar{t}]), \quad-\frac{\pi}{2}<\bar{t}<\frac{\pi}{2},
\end{aligned}
$$

is to be positive definite. Therefore, in the first case addressed in Eq. (A.6), Eq. (A.2) can be validated by studying the positive definiteness of $D^{\prime}(\bar{t})$ as follows:

$$
\begin{aligned}
D^{\prime}(\bar{t}) & =\cos \bar{t}+\frac{3}{4 N+1} \cos [(4 N+1) \bar{t}], \\
-\frac{\pi}{2} & <\bar{t}<\frac{\pi}{2} .
\end{aligned}
$$

To check the positive definiteness of $D^{\prime}$, the variations of $D^{\prime}$ at the interval $-\pi / 2<\bar{t}<\pi / 2$ for different values of $N$ are plotted in Figure A.1. It is seen that $D^{\prime}$ is positive; thus, Eq. (A.2) is valid for the first case in Eq. (A.6).

In the other case, Eq. (A.5) gives:

$$
\begin{aligned}
& u_{1}(t)=-\cos \left[\sqrt{1-\xi_{1}^{2}} t\right] e^{-\xi_{1} t}, \\
& u_{2}(t)=\frac{3}{4 N+1} \cos \left[(4 N+1) \sqrt{1-\xi_{1}^{2}} t\right] e^{-\xi_{1} t}, \\
& u_{1}(t=0)>0 .
\end{aligned}
$$

By replacing $t$ with $\overline{\bar{t}}$ defined below:

$$
(2 k+1) \pi+\overline{\bar{t}}=t \sqrt{1-\xi_{1}^{2}} .
$$

Eq. (A.14) and the second relation in Eq. (A.6) will lead to:

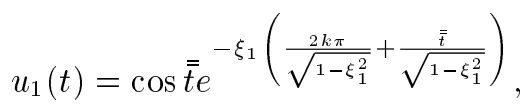

$$
\begin{aligned}
& u_{2}(t)=\frac{-3}{4 N+1} \cos [(4 N+1) \overline{\bar{t}}] e^{-\xi_{1}\left(\frac{2 k \pi}{\sqrt{1-\xi_{1}^{2}}}+\frac{\overline{\bar{t}}}{\sqrt{1-\xi_{1}^{2}}}\right)}, \\
& -\frac{\pi}{2}<\overline{\bar{t}}<\frac{\pi}{2} .
\end{aligned}
$$

The validity of Eq. (A.2) is then equivalent to the positive definiteness of $D^{\prime \prime}$ :

$$
\begin{aligned}
D^{\prime \prime}(\overline{\bar{t}}) & =\cos \overline{\bar{t}}+\frac{3}{4 N+1} \cos [(4 N+1) \overline{\bar{t}}], \\
-\frac{\pi}{2} & <\overline{\bar{t}}<\frac{\pi}{2},
\end{aligned}
$$

which is valid since Eq. (A.17) is conceptually identical to Eq. (A.13), and Figure A.1 shows that $D^{\prime \prime}>0$.

From simple geometry [74], it is apparent that reducing $u_{2}(t=0)$, i.e.:

$$
0<-u_{2}(t=0)<\frac{3 u_{1}(t=0)}{4 N+1},
$$

would lead to conservative responses; see Figure A.2. Consequently, the proof is complete, and Eq. (18) 


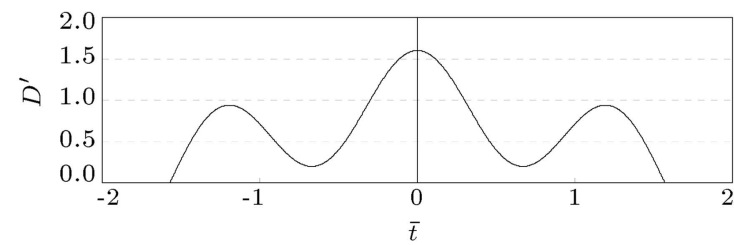

(a)

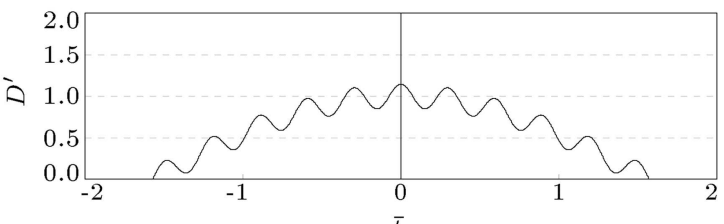

(c)

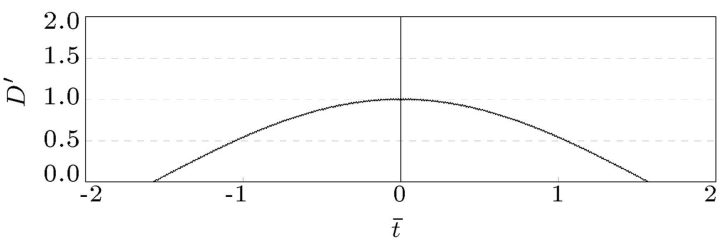

(e)

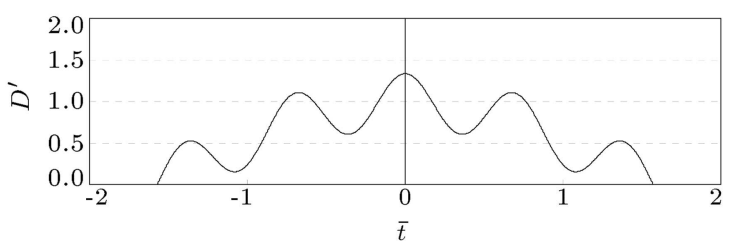

(b)

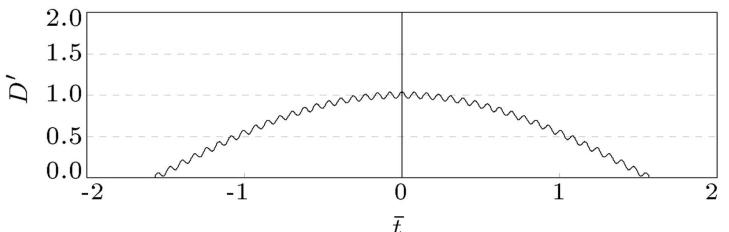

(d)

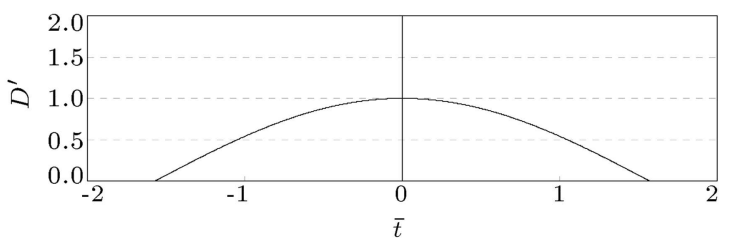

(f)

Figure A.1. Positive-definiteness of the $D^{\prime}$ defined in Eq. (A.13): (a) $N=1$, (b) $N=2$, (c) $N=5,($ d) $N=20,($ e) $N=100$, and (f) $N=10^{5}$.
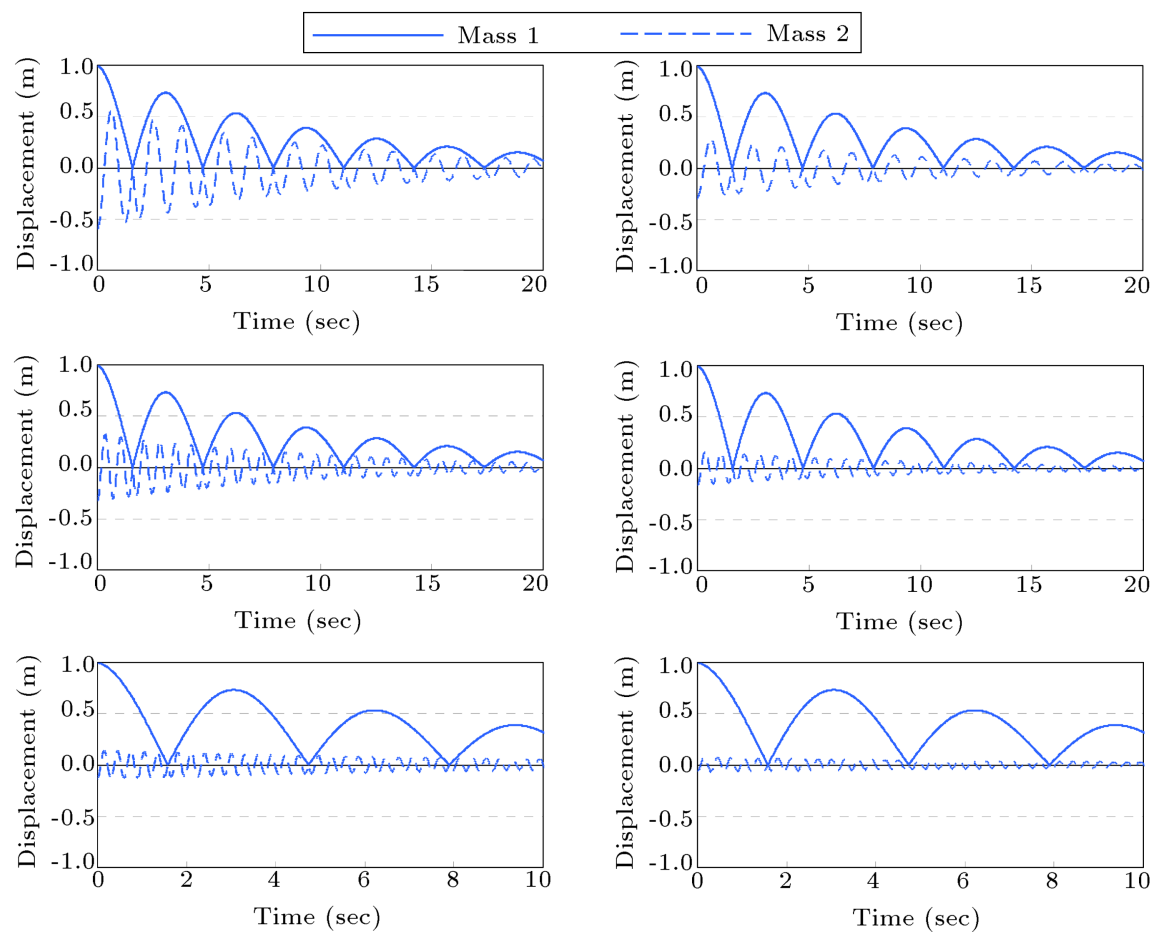

(a)

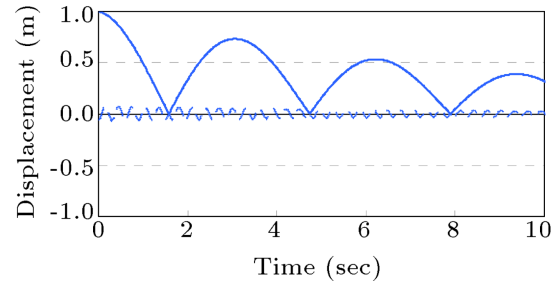

(b)

Figure A.2. Typical comparison between Eqs. (A.4) and (A.18) for potential collisions not at $u=0$ in the system proposed in Section $2\left(\xi_{1}=0.1, N=1,2,5\right):(\mathrm{a})-u_{2}(t=0)=3 u_{1}(t=0) / 4 N+1$ (Eq. (A.4)), and (b) $-u_{2}(t=0)=1.5 u_{1}(t=0) / 4 N+1$.

is a sufficient condition to prevent collisions only in the static equilibrium position. The conservativeness implied in Figures A.1 and A.2 (i.e., $D^{\prime}(\bar{t})$ is never zero at the interval $-\pi / 2<\bar{t}<\pi / 2)$ gives rise to questions about the origin of Eq. (18). As a brief response, attention should be paid to Figure A.3. Figure A.3. simply explains that, for small values of $\xi_{1}$, Eq. (18) is a sufficient condition against collisions not in the static equilibrium position in the system. 


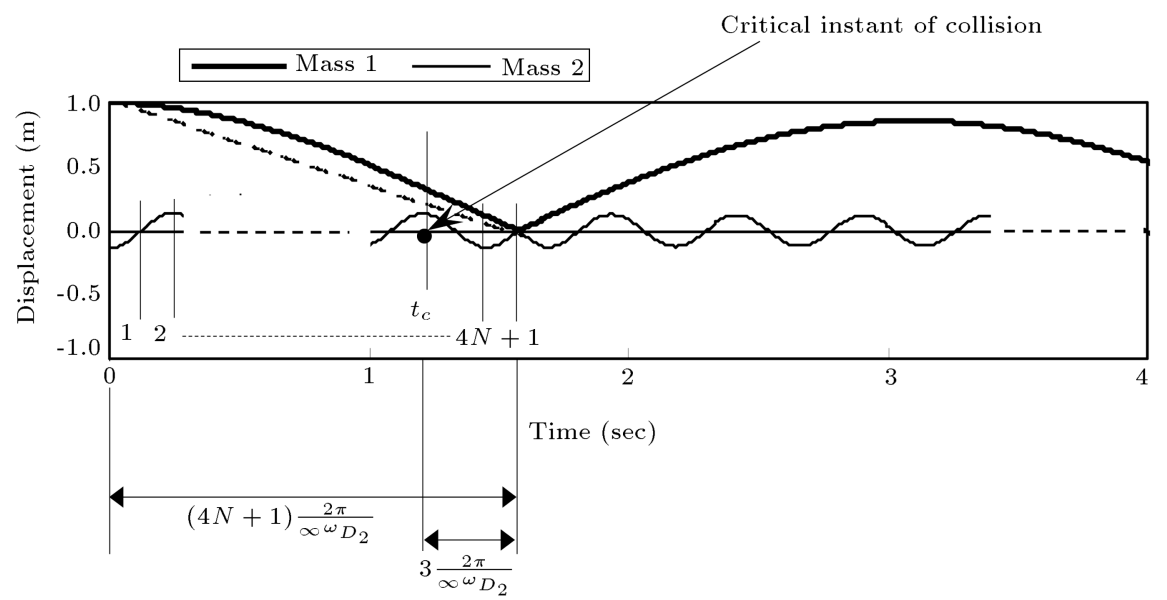

Figure A.3. Details of the most probable collision not in the static equilibrium position between masses of the system proposed in Section 2 when the damping is small.

\section{Biographies}

Aram Soroushian is an Assistant Professor at the Structural Engineering Research Center of the International Institute of Earthquake Engineering and Seismology (IIEES). He has obtained his BS, MS, and $\mathrm{PhD}$ degrees from University of Tehran, Shiraz University, and University of Tehran, respectively. The interests of Dr. Soroushian include computational mechanics, time history analysis, nonlinear behavior and analysis, structural dynamics, errors evaluation, and optimization.

Goodarz Ahmadi is a Clarkson Distinguished Professor, and Robert R. Hill Professor of Mechanical and Aeronautical Engineering at Clarkson University. He has obtained his MS and $\mathrm{PhD}$ degrees from Purdue University. He is a Fellow of ASME, ISME, and ISCE. Among his many awards, he was the recipient of the 2016 ASME Freeman Scholar Award. He was also elected as the "Chehreh Mandegar" (ever-lasting name) in Mechanical Engineering in 2003 .

He has four patents and authored three books and over 650 publications in archival journals. He also has made more than 1200 presentations including 20 plenary and keynotes at national and international technical conferences, and has given more than 160 invited talks and short courses at other institutions. He has been serving as the editor, editorial board, and/or editorial advisor board of fifteen international journals.

His research interests include multiphase flows, particle transport and deposition, turbulence, flow control, granular flows, air pollution, flow through porous and fractured media, random vibrations, and structural mechanics. His research has been supported by the National Science Foundation, the Environmental Protection Agency, Department of Energy, NASA, AFOSR, NYSTAR, GE, Corning, IBM, Xerox, Kodak, and Dura Pharmaceutical.

He has held many administrative positions at Clarkson University including the MAE Department Chair 1992-1995, the Associate Dean of Engineering 2003-2005, the Interim Vice Provost for Research 2004-2005, and the Dean of Engineering 2005-2015. Earlier, he served as the Dean of Engineering of Shiraz University.

Saeed Amiri received his MS degree in Earthquake Engineering from International Institute of Earthquake Engineering and Seismology (IIEES), Tehran, Iran in 2015. He holds a BS degree in Civil Engineering from Arak University, Iran. The title of his MS thesis is "On the performance of the existing recommendations for time step size selection in nonlinear analysis of conventional buildings". His research is focused on numerical methods in earthquake en gineering, reliability analysis, nonlinear dynamics, uncertainty quantification, and seismic performance of structures. 